\title{
The EXOSAT medium-energy slew survey catalog ${ }^{\star}$
}

\author{
A.P. Reynolds, A.N. Parmar, P.J. Hakala, A.M.T. Pollock, O.R. Williams, A. Peacock, and B.G. Taylor \\ Astrophysics Division, Space Science Department of ESA, ESTEC, P.O. Box 299, 2200 AG Noordwijk, The Netherlands
}

Received March 17; accepted July 16, 1998

\begin{abstract}
We present a catalog of X-ray sources observed during slew maneuvers by the Medium Energy Detector Array onboard the EXOSAT Observatory. The EXOSAT Medium Energy slew-survey catalog (EXMS) provides a unique record of the $1-8 \mathrm{keV} \mathrm{X}$-ray sky between 1983 and $1986.98 \%$ of the sky was observed, with $85 \%$ receiving an exposure of $>60$ s. 1210 sources were detected. By comparing these source positions with other catalogs, identifications are given for 992 detections (82\% of the sample). These identifications consist of 250 distinct objects, including 95 different X-ray binary systems, and 14 different AGN. A further 58 detections have multiple candidates, while 160 detections remain unidentified. Collimator transmission corrected $1-8 \mathrm{keV}$ count rates are given for the identified sources, together with raw count rates for the other detections. The construction of the EXMS and the checks performed to ensure the validity of the derived source properties are discussed. A publically available version of this catalog is maintained on the EXOSAT database and archive system (telnet://xray@exosat.estec.esa.nl).
\end{abstract}

Key words: astronomical data bases: miscellaneous catalogs - X-rays: general

\section{Introduction}

Sky surveys are of particular importance in high energy astronomy, where many sources exhibit irregular long-term variability which cannot be conveniently monitored by pointed observations. Observations conducted while maneuvering between targets can provide a substantial bonus to the scientific return of pointed missions

Send offprint requests to: A.P. Reynolds:

areynold@astro.estec .esa.nl

* Tables 3, 4 and 5 are only available in electronic form at the CDS via anonymous ftp to cdsarc.u-strasbg.fr (130.79.128.5) or via http://cdsweb.u-strasbg.fr/Abstract.html (e.g., Elvis et al. 1992). Such observations are complementary to the dedicated all-sky surveys conducted by scanning instruments such as the Uhuru, Ariel-V, HEAO A-1, and ROSAT bright source catalogs (Forman et al. 1978; Warwick et al. 1981; McHardy et al. 1981; Wood et al. 1984; Voges et al. 1996). Here we report the second major X-ray slew survey, derived from observations made by the European Space Agency's EXOSAT X-ray astronomy satellite (White \& Peacock 1988).

EXOSAT performed 1780 pointed observations of a wide variety of objects between 1983 June and 1986 April. The $90 \mathrm{hr}$ orbit had an apogee of $190000 \mathrm{~km}$ and perigee of $350 \mathrm{~km}$, with the science payload operated when the satellite was above the Earth's radiation belts at $50000 \mathrm{~km}$. This allowed uninterrupted observations of up to $76 \mathrm{hr}$ duration. The satellite was three axis stabilized, and at any given time about half the sky could be viewed. X-ray sources were simultaneously observed with up to 4 coaligned instruments. Two Channel Multiplier Array detectors (CMA; de Korte et al. 1981) each at the focus of an X-ray mirror provided images in the low-energy $(0.04-2.0 \mathrm{keV})$ energy range, while the Medium Energy Detector Array (ME; Turner et al. 1981) and the Gas Scintillation Proportional Counter (GSPC; Peacock et al. 1981) covered the $1-50 \mathrm{keV}$ and $2-35 \mathrm{keV}$ energy ranges, respectively. In addition to the the pointed observations, a series of slews along parts of the galactic plane were performed (Warwick et al. 1985, 1988).

When EXOSAT manoeuvred between targets the ME and GSPC instruments were usually operated in order to search for new sources, monitor known ones, and to measure background counting rates. The relatively slow manoeuvre rates of EXOSAT (either 42,85 , or $170^{\circ} \mathrm{hr}^{-1}$ ), together with a good knowledge of the pointing direction during slews, allows the construction of a catalog with high sky-coverage and sensitivity. Slew manoeuvres were usually performed in three stages or legs, rather than along the great circles directly between sources. First there would be a slew to place the instruments' pointing axis $90^{\circ}$ from the Sun (at a $\beta$ angle of $90^{\circ}$ ), followed by a slew along 


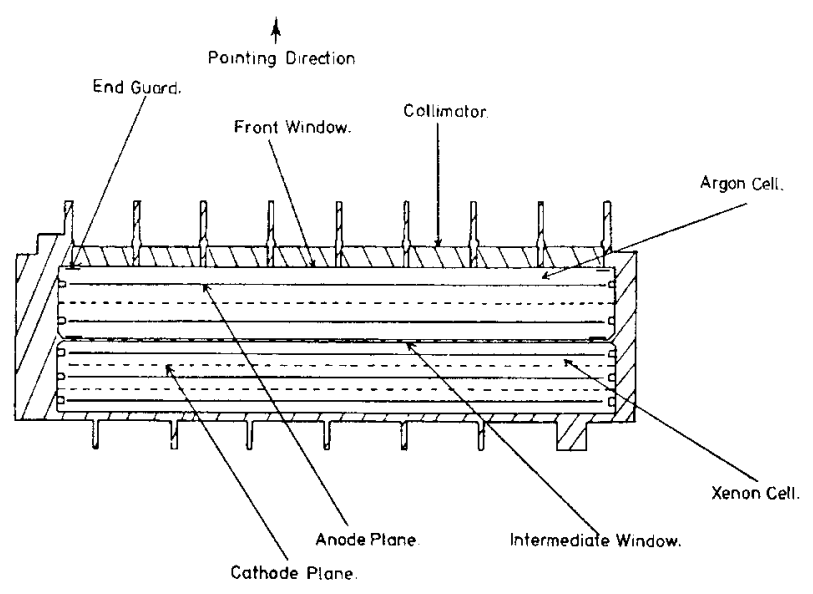

Fig. 1. Cross-section of an ME detector

the $\beta=90^{\circ}$ line and then a final slew off the $\beta=90^{\circ}$ line to the new pointing position. Apart from maximizing the efficiency of solar power collection, this procedure resulted in greater sky coverage than if the slews were along connecting great circles. During EXOSAT operations the ME slew data were routinely checked for the presence of X-ray sources. This led to the discovery of four previously unknown X-ray sources, all of which were found to be X-ray binaries (see White \& Peacock 1988).

\section{The Medium Energy detector array}

\subsection{The detectors}

The performance of the ME is summarized in Table 1. The ME comprised 8 individual detectors, each of which consisted of an $\mathrm{Ar} / \mathrm{CO}_{2}$ and a $\mathrm{Xe} / \mathrm{CO}_{2}$ gas filled multiwire proportional counter separated by a $1.5 \mathrm{~mm}$ thick Be intermediate window (Fig. 1). An X-ray collimator, made from lead-glass microchannel plates was mounted in front of each detector. Anticoincidence and pulse rise time techniques were used to reduce the particle background. The ME operated well throughout the mission with one of the detectors failing on 1985 August 20. Problems with detector breakdown at the start of the mission were solved by operating the Ar counters at a lower overall gain setting. This resulted in pulse-height analyzer (PHA) channel 128 corresponding to an energy of $\sim 50 \mathrm{keV}$.

\subsection{Background}

The ME background was usually stable with time and dominated by particle-induced events from the solar wind and events from the radioactive lines of residual Plutonium in the Be windows and detector bodies. The contribution of the extragalactic X-ray background was $<1 \%$ of the total Ar background counting
Table 1. Properties of the EXOSAT ME

\begin{tabular}{ll}
\hline Geometric Area (8 detectors) & $1600 \mathrm{~cm}^{2}$ \\
Field of view (FWHM) & $\sim 45^{\prime} \times 45^{\prime}$ \\
Ar counter energy range & $1-20 \mathrm{keV}$ \\
Xe counter energy range & $5-50 \mathrm{keV}$ \\
Number of PHA channels & $128+128$ (Ar and Xe) \\
Energy Resolution (Ar) & $49 / E\left(\mathrm{keV}^{0.5} \% \mathrm{FWHM}\right.$ \\
$1-8 \mathrm{keV}$ Background & 21 counts s \\
(8 detectors) & \\
\hline
\end{tabular}

rate. After anticoincidence rejection of particle-induced events the typical $1-8 \mathrm{keV}$ Ar background count rates was $3.8 \mathrm{~s}^{-1}$ detector $^{-1}$. Occasional background flares occurred simultaneously in some or all of the detectors and were caused by enhancements in the solar wind. For normal observations longer than $\sim 5000 \mathrm{~s}$, the ME was limited by systematic effects in the background subtraction to detections of $\gtrsim 0.510^{-11} \mathrm{erg} \mathrm{cm}^{-2} \mathrm{~s}^{-1}$.

\subsection{Field of view}

The ME field of view (FOV) was defined by collimators which had a rectangular aperture with an average fullwidth half-maximum (FWHM) of $45^{\prime}$ and a flat top of $\sim 7^{\prime}$ (Fig. 2). Details are to be found in Gottwald (1984) and Kuulkers (1995). Figure 2 shows a labeled schematic model of the collimator response. The 8 detectors were grouped into four pairs or "quadrants", each of which could be offset by up to $120^{\prime}$ from the aligned position. For most targets, two quadrants (one half of the ME) would be aligned and pointed at the target, while the remaining quadrants would be offset and pointed at two adjacent regions of sky in order to monitor the background counting rate. For bright sources, where background subtraction is not so critical, the ME could be operated with all four quadrants observing the source. The orientations of the quadrants are stored in the spacecraft pointing files, which were updated every $60 \mathrm{~s}$.

\subsection{Additional slew observations}

As well as the slew manoevers required to move between scheduled pointings, EXOSAT performed a series of scans along parts of the galactic plane as part of the scientific program. The results are presented in Warwick et al. $(1985,1988)$ and are not included in the EXMS.

\section{The EXOSAT ME slew survey catalog (EXMS)}

\subsection{Data selection}

As part of the routine data processing during the EXOSAT post-operational phase, slew lightcurves 


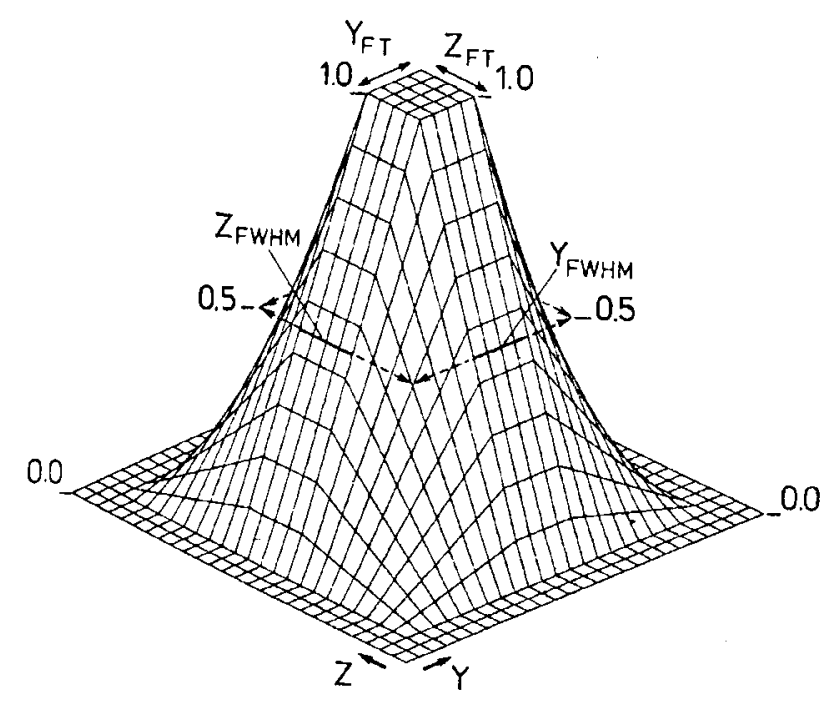

Fig. 2. A schematic model of an idealized ME collimator response (Kuulkers 1995). Slew directions were always parallel to the $Y$ or $Z$ axes. The flat top $\left(Y_{\mathrm{F}} T, Z_{\mathrm{F}} T\right)$ to the collimator response had approximately equal sides of length 7 arcmin. The numbers indicate the relative transmission

covering the energy ranges $1-8 \mathrm{keV}$ and $10-18 \mathrm{keV}$ were systematically produced.

If data were available for individual detectors or quadrants, then three lightcurves were produced for each energy range and time interval; one for the two aligned quadrants and one for each of the offset quadrants (since they pointed in different directions on the sky). If data were only available for each half-array, then separate lightcurves for the aligned and offset quadrants were produced for each energy range. In this case, a source detected in an offset quadrant could not always be unambiguously located on the sky, since the counts may have arisen from one of two separate locations. In the cases when a single data stream covering the entire detector was selected, only a single set of lightcurves covering both the aligned and offset quadrants was produced. The time taken for a source to pass through a FOV is dependent on the slew rate, which was nominally $42^{\circ} \mathrm{hr}^{-1}$, with two faster speeds of 85 and $170^{\circ} \mathrm{hr}^{-1}$ also used. At the slowest rate, a source would take $135 \mathrm{~s}$ to pass completely through the FOV, from zero response to zero response. The $1-8 \mathrm{keV}$ energy range was chosen since this is where the residual background in the ME was lowest and the sensitivity highest for a source with a typical X-ray spectrum. The $10-18 \mathrm{keV}$ energy range was chosen to sample the solar particle induced background counting rate; the contribution of most $\mathrm{X}$-ray sources in this energy range being small. The slew lightcurves were accumulated with a binning time of $10 \mathrm{~s}$. This was chosen to allow a uniform set of lightcurves to be produced, since all the ME data configurations provided spectra with $10 \mathrm{~s}$ or faster time resolution, while allowing the collimator profile to be adequately sampled while slewing.

\subsection{Exposure map}

Data from a total of 1765 slews, $>99 \%$ of the total were processed. Figure 3 is an exposure map of the data used to construct this catalog. The map was generated by first calculating the region of sky swept out by each quadrant on the celestial sphere, using the positional information in the spacecraft pointing files. The sky was then divided into cells of width $0.25^{\circ}$, and each cell was assigned an effective exposure, dependent on the slew rate and the distance of the cell from the centroid of the collimator. Individual cells were often slewed over repeatedly, especially near the ecliptic poles, and the effective exposures were updated for each subsequent slew over a given cell. These values were then converted into contours and plotted in a Hammer-Aitoff projection using Galactic coordinates. The efficiency of sky coverage was high: $98 \%$ of the sky was slewed over at least once, while $85 \%$ received an exposure of $>60 \mathrm{~s}$. The few regions of the sky which were not slewed over at all, or had exposures $<3 \mathrm{~s}$, or where there is no pointing data, are indicated in black. All other areas received exposures between $3 \mathrm{~s}$ and $6000 \mathrm{~s}$. This compares favorably with the exposure obtained by the Einstein slew survey, where useful sensitivity was only obtained for $50 \%$ of the sky (Elvis et al. 1992). As in the Einstein slew survey, the areas of sky which received the highest exposure are near the ecliptic poles, but unlike the latter the Galactic plane is also well-sampled, since there was no requirement to avoid slewing across bright sources. This means that the slew survey (hereafter EXMS) is expected to be rich in compact X-ray sources.

A schematic diagram of a typical three-legged slew is shown in Fig. 4. The direction of slew is always parallel to one edge of the rectangular FOV. In Fig. 5 the region of the sky swept out by a real three-legged slew is shown. In the first, second, and third legs, the FOVs swept out two, three and two strips of sky, respectively. The differing paths swept out by the aligned and offset quadrants are shown, together with the positions of the eight detected sources.

\subsection{Source detection}

To illustrate the quality and type of data available in the EXMS, Fig. 6 shows representative $1-8 \mathrm{keV}$ lightcurves for a slew across a crowded region of sky (the same slew as illustrated in Fig. 5). The three panels show lightcurves for the aligned and two offset quadrants. One source (4U 1627-673) is seen in the two offset quadrants at different times (separated by $240 \mathrm{~s}$ ). All count rates are normalized to $\mathrm{s}^{-1}$ half $^{-1}$ (4 detectors), but no correction for 


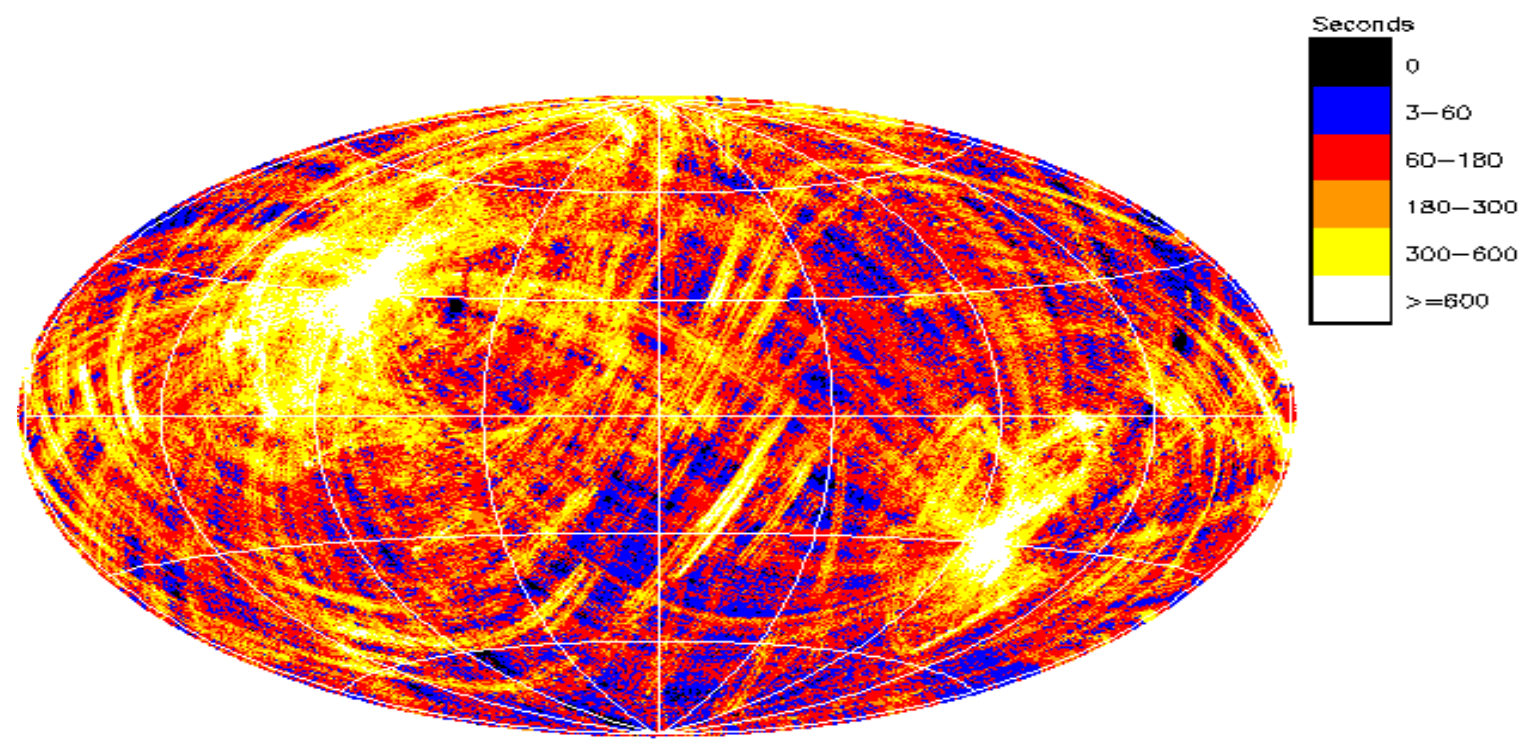

Fig. 3. Exposure map for the complete EXMS catalog in galactic coordinates. Areas which were never slewed over, or had exposures of $<3 \mathrm{~s}$ are shaded black. All colored regions received an exposure of $\geq 3 \mathrm{~s}$. Areas colored white received exposures $>600$ s. $98 \%$ of the sky was slewed over at least once and $85 \%$ of the sky received an exposure of $>60 \mathrm{~s}$

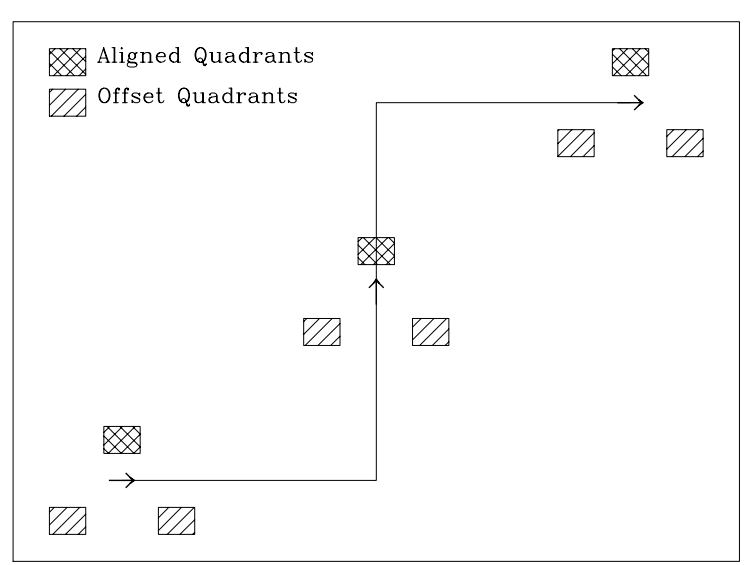

Fig. 4. Schematic diagram of a three-legged slew, with the four quadrants of the ME arranged in the usual combination of two aligned and two offset. The squares indicate the FOVs of each quadrant and are not to scale. Slew directions were always parallel to one edge of the FOV

collimator losses has been applied. The lightcurves are not background subtracted and the nominal background counting rate of $\sim 15 \mathrm{~s}^{-1}$ half $^{-1}$ is visible whenever there are no sources in the FOV. The 3 slew legs can be seen in the lightcurves since there are missing data between the slew legs (just after $16 \mathrm{hr}$ and just before $16.6 \mathrm{hr}$ ). This is due to data from contiguous intervals of less than $5 \mathrm{~min}$ duration not being stored on EXOSAT data tapes.
The slew lightcurves were systematically searched for sources passing through the FOV by modeling the observed time-dependent $1-8 \mathrm{keV}$ count rate, $R_{1-8}$, by:

$$
R_{1-8}(T)=S H\left(T-T_{\mathrm{s}}\right)+k R_{10-18}(T) .
$$

The source term depends on the source count rate, $S$, the time of transit through the collimator center, $T_{\mathrm{s}}$, and the collimator profile $H\left(T-T_{\mathrm{s}}\right)$ determined by the slew rate. The $1-8 \mathrm{keV}$ background count rate was assumed to be linearly related through a constant, $k$, to the $10-18 \mathrm{keV}$ count rate, $R_{10-18}$. This was verified by inspection of the lightcurves which showed that only sources with peak count rates $\gtrsim 100 \mathrm{~s}^{-1}$ were detected in the $10-18 \mathrm{keV}$ energy range. This approach is successful in excluding the type of background events illustrated in the upper panel of Fig. 6, in which short-term increases in the solar particle flux can mimic the intensity profile of an X-ray source.

For a fixed $T_{\mathrm{s}}$ best-fit parameters $S$ and $k$ were determined by maximum likelihood. Candidate detections were initially identified in a coarse search in $T_{\mathrm{s}}$, after which parameters and their uncertainties were precisely calculated. The transit of some strong sources can be located to $<0.1 \mathrm{~s}$. Occasionally, it was necessary to fit two sources simultaneously. The limiting $1-8 \mathrm{keV}$ sensitivity, when no confusing sources are present, is estimated to be $310^{-11} \mathrm{erg} \mathrm{cm}^{-2} \mathrm{~s}^{-1}$ at the slowest slew rate. The source best-fit locations and error boxes were reconstructed using the values of $T_{\mathrm{s}}$ and $\Delta T_{\mathrm{S}}$ by parabolic interpolation 


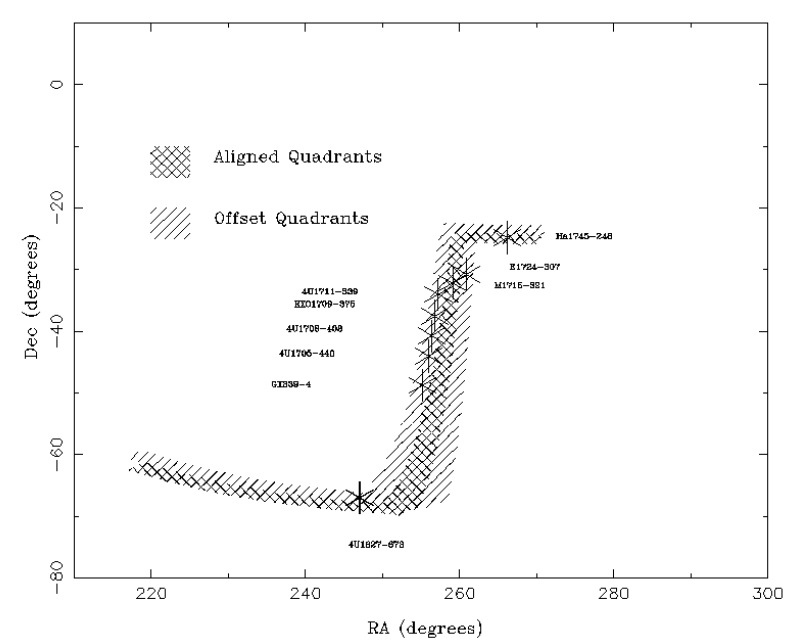

Fig. 5. The "footprint" of a representative three-legged slew showing the area of sky observed. The hatched and crosshatched areas show the area covered by the offset and aligned quadrants, respectively. The start of the slew was in the lower left corner. The variation in the width of the footprint results from the projection onto equatorial coordinates. The positions of the sources identified in Fig. 6 are marked with stars

from the positions contained in the pointing files. A total of 1210 detections were made in this way and are shown in galactic coordinates in Fig. 9.

If the fit to the collimator profile is formally acceptable, the time of maximum intensity corresponds to the position of the quadrant when the source passed through its midline. Hence, source positions can be well localized in the direction of slew, but the position perpendicular to the slew axis is constrained only by the width of the FOV, unless assumptions about the source intensity are made. This means that, for the majority of sources, the uncertainty regions are narrow in the slew direction and much broader perpendicular to it.

The mean length of the narrow side of the 1210 uncertainty regions is $(6.30 \pm 0.12)^{\prime}$; (all errors are given at $68 \%$ confidence). In Fig. 7 a schematic diagram of a typical EXMS uncertainty region is shown defining the different offsets which are referred to later. In Fig. 8 the lengths of the narrow sides are plotted against uncorrected source counts for the 1210 detections. This demonstrates that the brightest detections tend to have the narrowest uncertainty regions, as expected. Since these regions are constructed under the assumption of a constant source intensity, it is to be expected that some identifications will lie outside their formal uncertainty region (see Fig. 7). Also seen in Fig. 8 is the effect of the different slew rates on the width of the uncertainty region for a given count rate, leading to three distinct clusterings of points, superimposed on the scatter caused mainly by source variability.
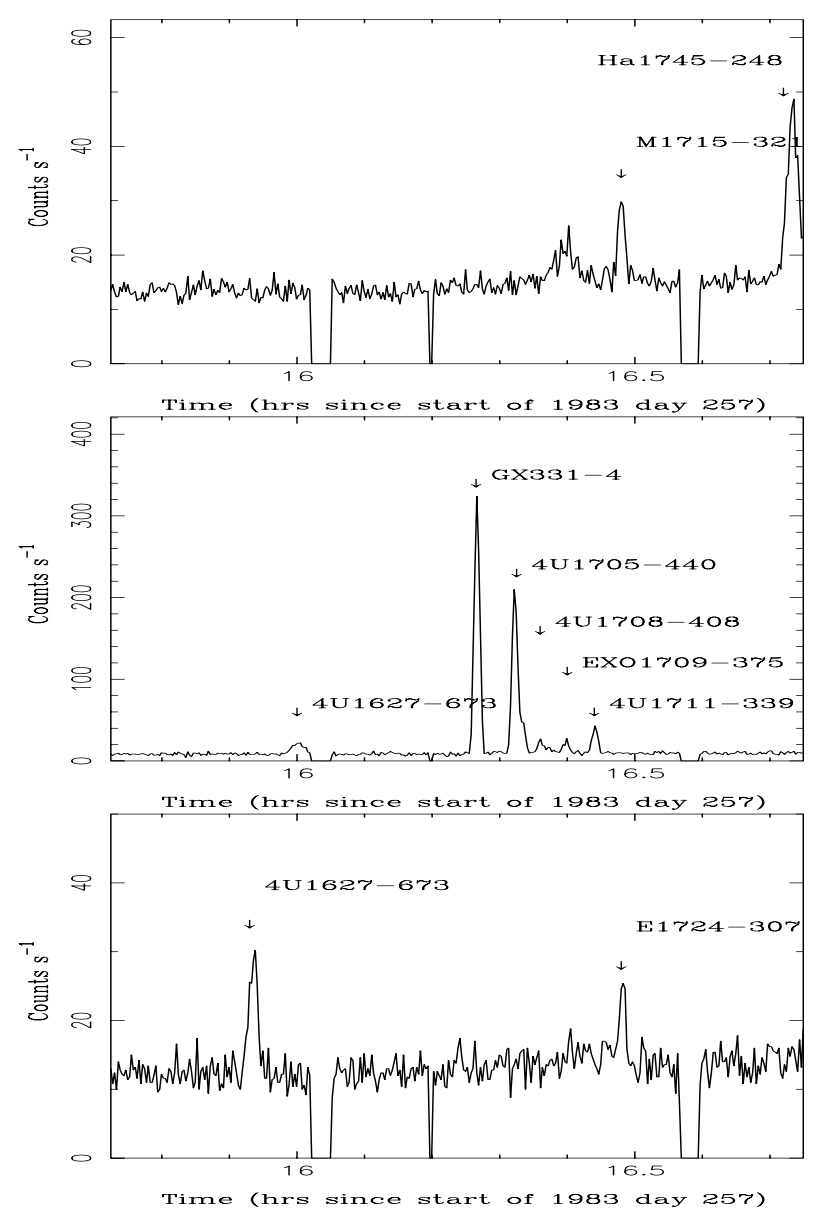

Fig. 6. Lightcurves obtained during a slew across a crowded region of sky, obtained from the two aligned quadrants (upper panel) and the two offset quadrants (middle and lower panels). No correction for collimator losses has been applied. Source identifications are given. The count enhancement at around $16.4 \mathrm{hr}$ in the aligned quadrants is due to increased solar activity. The slew rates were $42^{\circ} \mathrm{hr}^{-1}, 85^{\circ} \mathrm{hr}^{-1}$ and $42^{\circ} \mathrm{hr}^{-1}$ during the first, second, and third slew legs, respectively. The intervals of missing data correspond to the breaks between slew legs. Note that $4 \mathrm{U} 1627-673$ was detected in both offset quadrants separated by $240 \mathrm{~s}$

\subsection{Source indentification}

Where possible, sources responsible for the 1210 detections have been identified by correlation against previous catalogs. The procedure is to determine all the sources which fall within the $99.7 \%$ confidence uncertainty region for a particular detection. This may include entries from more than one catalog. A preferred identification is then made using a hierarchical selection scheme. The following catalogs were used to generate possible identifications, and are listed in hierarchical order:

- XRBCAT: A catalog of X-ray Binaries derived from van Paradijs (1995).

- RITTER: A catalog of cataclysmic variables, X-ray binaries and related objects, derived from Ritter (1990). 


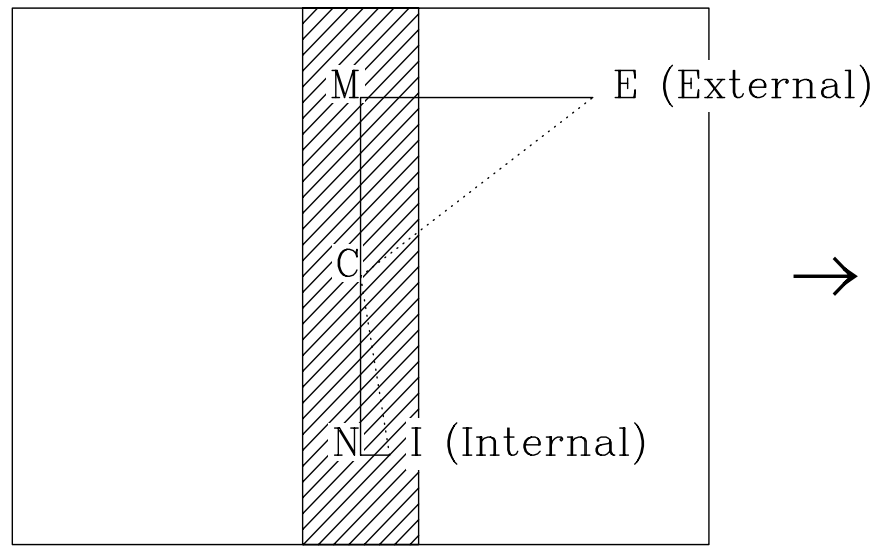

Fig. 7. Schematic diagram of a formal EXMS uncertainty region, shown as a hatched rectangle within the rectangular FOV. The arrow shows the slew direction. The source positions $\mathrm{I}$ and $\mathrm{E}$ are internal and external to the uncertainty region, respectively. $\mathrm{C}$ is the uncertainty region centroid. EC and IC are the distances between sources $\mathrm{E}$ and I and the centroid. EM and IN are the parallel, or vector, offsets in the direction parallel to the slew. $\mathrm{CM}$ and $\mathrm{CN}$ are the distances which determine the correction for collimator transmission

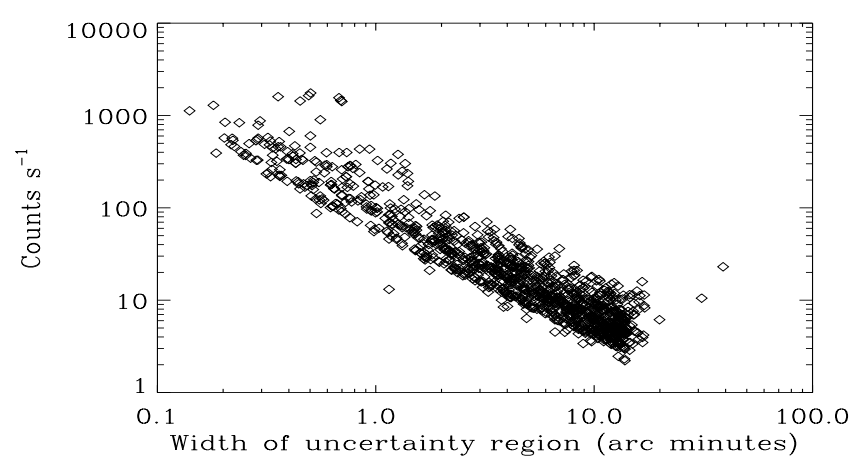

Fig. 8. Plot of the dimensions of the short sides of 1210 uncertainty regions against source count rate. The clustering of the data points into three distinct lines is a consequence of the three different slew rates, the slowest of which allowed sources to be localized most precisely

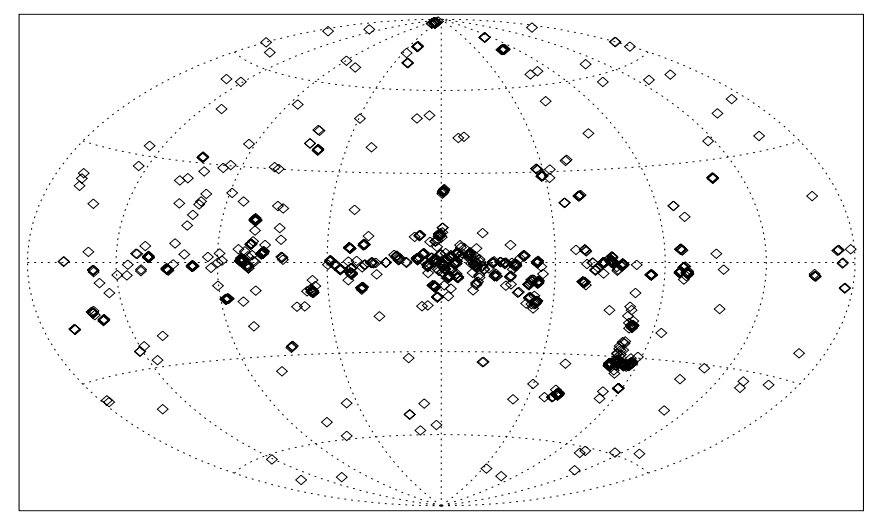

Fig. 9. The 1210 EXMS detections in galactic coordinates. The predominance of galactic plane and Magellanic cloud sources is evident
- VSTARS: The General Catalog of Variable Stars, derived from the 69th name list of variable stars (Kholopov et al. 1989).

- XRAY: A master catalog containing selected parameters from all X-ray catalogs present on the online system, including HEAO-1, Einstein, EXOSAT and ROSAT source catalogs.

- VERON96: Derived from the 7th edition of the Catalog of Quasars and Active Galactic Nuclei by Veron-Cetty \& Veron (1996).

- RADIO: A master catalog containing selected parameters from several radio source catalogs.

At the end of this search, a given detection would either have produced no identification, a single identification, or multiple candidates. In order to ensure that these identifications were always known X-ray emitters, a further search was made against the ROSAT All-Sky Survey Bright Source catalog (RASSBSC; Voges et al. 1996, 1998), which had not been incorporated into the XRAY catalog at the time of the initial search. The RASSBSC was used to validate all proposed identifications and to search for any additional candidates which were not present in the other catalogs. Consequently, after cross-checking against the RASSBSC, many candidate identifications derived from the VSTAR, RADIO and VERON96 catalogs were discarded. Known transient $\mathrm{X}$-ray sources, and extended X-ray objects (which do not appear in the RASSBSC) are retained.

The sources found in the above searches are all within the formal uncertainty regions. However, apart from these "internal" sources, attention was also paid to any bright, time variable and/or extended sources whose derived positions were outside the formal uncertainty regions, but which still passed within the FOV during a slew. Such sources, typically X-ray binaries or supernova remnants, may well have incorrectly determined uncertainty regions due to time-variability, or their extended nature. In practise, whenever this occured, there was little doubt that the detection was due to the "external" source, since there was either no candidate in the uncertainty region, or a source not known to be a strong X-ray emitter.

Some sources are considered confused; i.e., while it is likely that the majority of counts originate from the object proposed as the identification, the RASSBSC indicates that at least one fainter source was present in the uncertainty region. The fainter source may therefore contribute to the signal. The criterion used for classifying a source as confused is that the brightest RASSBSC source must be at least four times brighter than any other source within the uncertainty region. Where this is not the case (ie where the RASSBSC sources differ by less than a factor of four in brightness) no single identification is proposed. In this case we assume that the brightest RASSBSC source (based on 0.1-2.4 keV ROSAT Position Sensitive Proportional Counter countrates) is also the brightest in the $1-8 \mathrm{keV}$ EXMS range. 
Table 2. Counterpart statistics and flag values

\begin{tabular}{lllrll}
\hline Flag & $N_{\text {samp }}$ & $\%$ & $N_{\text {int }}{ }^{a}$ & $N_{\text {ext }}{ }^{b}$ & Comment \\
\hline 4 & 617 & $51 \%$ & 1 & 0 & Internal \\
3 & 355 & $29 \%$ & 0 & 1 & External \\
2 & 20 & $2 \%$ & $>1$ & 0 & Confused \\
1 & 58 & $5 \%$ & $>1$ & 0 & Multiple \\
0 & 160 & $13 \%$ & 0 & 0 & Unidentified \\
\hline
\end{tabular}

${ }^{a}$ Number of sources within the uncertainty region.

${ }^{b}$ Number of sources outside the uncertainty region, but within the FOV.

Flags have been assigned to all entries to distinguish the type of identification; they may also be considered as approximate quality flags. This scheme is summarized in Table 2, together with the numbers and percentages of sources in each category. $51 \%$ of the entries in the slew survey are identified with confidence - "internal" sources where the most likely X-ray source also lies within the uncertainty region. A further $29 \%$ of entries consist of identified sources which lie outside their formal uncertainty regions. Although these identifications are considered secure, the derived count rates are likely to contain larger errors than the internal sample, due to the error in the initial fit to the lightcurve (this problem is particularly severe for $\mathrm{X}$-ray pulsars where the duty cycle of the pulsations may be $\$ 50 \%$ ). The mean reduced $\chi^{2}$ for the internal sample is 1.3 , whereas that for the external sources it is 48 . The majority of these identifications are X-ray binaries. Hence, $82 \%$ of the entries are given single identifications, similar to the identified fraction in the Einstein slew survey. A further $2 \%$ of sources are "confused" - there is a fainter source (at least four times fainter, based on RASSBSC count rates) present in the uncertainty bound.

A further $5 \%$ of entries consist of cases where multiple sources of similar brightness lie within the formal uncertainty region. $13 \%$ of the entries cannot be identified with any known X-ray emitters, either because there are no $\mathrm{X}$-ray emitters in the vicinity or there is significant doubt about the likely identification. In the case of the $82 \%$ of entries where one identification is proposed (sources with Flags of 2, 3, and 4), the count rates given in Table 3 are corrected for collimator transmission. This is achieved by calculating the offset of the source (based on its catalog coordinates) from the centroid of the detector (see line $\mathrm{CM}$ in Fig. 7). The correction is proportional to this offset, except for the small "flat-top" at the center of the FOV (see Fig. 2). In addition, count rates are normalized to count $\mathrm{s}^{-1}$ half ${ }^{-1}$, ensuring consistency with the count rates present in the ME database.

Information on the 992 sources with single identifications is presented in Table 3 . The first column is a designation in the form EXMS BHHMM+DDd, where HHMM and DDd are the RA and Decl. of the uncertainty region centroid in epoch 1950 coordinates. (Note: in order to maximise the usefulness of Tables 3,4 and 5, all coordinates therein are presented for epoch 2000, although all prior catalog searches and computations were performed using epoch 1950 coordinates).

Most detections have a unique EXMS classification. In cases where the classification is not unique, designators in the form (A), (B), etc are appended. In order to usefully group identifications, detections have been ordered in RA of the proposed candidate. Detections with the same coordinate (but unique designator) may be split between tables depending on the category of the identification. In cases where an identified source was observed more than once, the entries are additionally ordered by detection time. The format for the time entries is YYYY/DDD $\mathrm{HH}: \mathrm{MM}$, indicating an observation at MM minutes after HH hours on day DDD of year YYYY. The proposed identifications are given (truncated to 18 characters), followed by a "type" string which gives the first three letters of the catalog from which the identification was drawn (see the list of catalogs above), or the object category. The object categories are XRB: X-ray binaries; SNR: supernova remnant; CLU: cluster of galaxies; and AGN: active galactic nucleus. In this context we adopt the widest possible meaning of the term AGN, including Seyfert galaxies, BL Lacertae objects, QSOs, quasars, Radio Galaxies and Optical Violent Variables (OVVs). The next parameters are the collimator corrected count rate, together with the $\chi^{2}$ of the fit to the light curve and the number of degrees of freedom (dof). Large values of $\chi^{2} /$ dof may indicate source variability. Parameter $\lambda$ is the likelihood detection statistic which is the difference in the logarithm of the likelihood between the best-fit and null-hypothesis models with no source present. In the null-hypothesis, $2 \lambda$ is expected to be distributed as $\chi^{2}$. Flag values are given under the column labelled "F", (ranging between 2 and 4 for this sample). Finally, the collimator distances, $D$, (CM in Fig. 7) are given in arc minutes. Detections with collimator distances $\gtrsim 30^{\prime}$ may contain large errors in their corrected count rates and should be treated with caution. Remarks on individual entries are given in Sect. 3.5.

The 58 detections with multiple candidates $($ Flag $=1)$ are summarized in Table 4 . The names of the proposed candidates are stored in a string, the first 20 characters of which are printed in Table 4 , together with the number of possible identifications, $N_{\text {mult }}$. The count rates given are raw values, uncorrected for collimator transmission. More information on the proposed candidates is given in Sect. 3.5.

Table 5 contains information on the 160 unidentified detections $($ Flag $=0)$. In this case, in addition to the coordinates of the centroid of the uncertainty region, positions of the four bounding corners are also given (in decimal format for brevity), under columns labelled C1 to C4. Again, the count rates are uncorrected for collimator transmission. 


\subsection{Remarks on individual catalog entries}

Comments are provided for individual catalog entries, referenced by their EXMS designations (see Tables 3-5), below.

EXMS B0016 - 722 - AQ Tuc lies within the uncertainty region and is within $10^{\prime}$ of $5 \mathrm{X}$-ray sources. Globular cluster 47 Tuc is nearby.

EXMS B0029 - 841 - Four PKS sources lie within the uncertainty region, but none are cataloged X-ray emitters.

EXMS B0110+649 - The XRB 2S 0114+65 is nearby.

EXMS B0113 - 417 - UV Phe lies within the uncertainty region, but is not a cataloged X-ray emitter. The RASSBSC source adopted as the identification is consistent with the location of a cataloged X-ray emitting Seyfert galaxy.

EXMS B0114+005 - 1RXSJ011704.2+000025 may be associated with E0114.4-0015, a Seyfert 1.

EXMS B0121+341 - NGC 513 lies within the uncertainty region. It is not a cataloged X-ray emitter but is bright and close.

EXMS B0245 - 420 - DK Eri lies within the uncertainty region, but is not a cataloged X-ray emitter.

EXMS B0250+415 - Two X-ray counterparts of NGC 1129. Multiple entries are $1 \mathrm{H} 0251+414$ and IPC $025113+41$.

EXMS B0250+417 - $1 \mathrm{H} 0251+414$ and IPC025113+41 are two X-ray counterparts of NGC 1129.

EXMS B0250+418 - see above.

EXMS B0252 - 417 - PKS 0252-41 lies within the uncertainty region, but is not a cataloged X-ray emitter.

EXMS B0252+415 - an unidentified source possibly associated with the above object. Another unidentified source, EXMS B0252+411, lies less than one degree away.

EXMS B0252+411 — see above.

EXMS B0309 - 317 - PKS 0309-31 lies within the uncertainty region, but is not a cataloged X-ray emitter.

EXMS B0310+411 - May be NGC 1275 or Mrk 1073.

EXMS B0402 - 654 - PKS 0403-65 lies within the uncertainty region but is not a cataloged X-ray emitter.

EXMS B0431 - 613B - PKS 0429-61 lies within the uncertainty region, but is not a cataloged X-ray emitter.

EXMS B0439 - 129 - PKS 0436-129 lies within the uncertainty region but is not a cataloged X-ray emitter.

EXMS B0446+447-4C +44.12 and $4 \mathrm{C}+44.13$, cataloged X-ray emitting sources in $3 \mathrm{C} 129$, lie within the uncertainty region.

EXMS B0525 - 329 - MS 05267-3301 lies within the uncertainty region, but is not a cataloged X-ray emitter.
EXMS B0529 - 650 - The proposed RASSBSC identification is near the transient X-ray binary EXO 53109-66.

EXMS B0559 - 664 - possibility of confusion with A $0535-668$. Four RASSBSC sources within uncertainty region.

EXMS B0532 - 664B - see above.

EXMS B0533 - 663B - see above.

EXMS B0533 - 662A - see above.

EXMS B0534 - 657 - see above.

EXMS B0537 - 713 - Probably an LMC object; LMC $\mathrm{X}-1$ and $\mathrm{X}-2$ were both slewed over near the detection time, although neither fell within the uncertainty region, but the raw (and collimator corrected) count rate is too high for either. PKS 0531-71 lies within the uncertainty region, but is not a cataloged X-ray emitter.

EXMS B0601 - 701 - an unidentified source less than one degree from another unidentified source, EXMS B0606-697.

EXMS B0606 - 697 - see above.

EXMS B0607 - 712 - PKS 0611-71 lies within the uncertainty region, but is not a cataloged X-ray emitter. An unidentified source, EXMS B0610-714, lies less than one degree away.

EXMS B0610 - 714 - see above.

EXMS B0613+228 - Two obscure radio sources, PKS 0615+22 and $4 \mathrm{C}+22.14$, neither cataloged as an $\mathrm{X}$-ray emitter.

EXMS B0613+229 - 4C +22.14 lies within the uncertainty region, but is not a cataloged X-ray emitter.

EXMS B0629 - 713 — an unidentified source less than one degree from another unidentified source, EXMS B0640 - 715 .

EXMS B0640 - 715 - see above.

EXMS B0648 - 698 - PKS 0650-70 lies within the uncertainty region, but is not a cataloged X-ray emitter.

EXMS B0654 - 559 - PKS 0649-55 lies within the uncertainty region, but is not a cataloged X-ray emitter.

EXMS B0655 - 707 - an unidentified source less than one degree from another unidentified source, EXMS B0656 - 697.

EXMS B0656 - 697 - see above.

EXMS B0658+753 - 4C +74.12 lies within the uncertainty region, but is not a cataloged X-ray emitter.

EXMS B0821 - 425A - 1ES 0821-426 is a cataloged $\mathrm{X}$-ray source.

EXMS B0821 - 425B - an unidentified source which might be associated with the above, although it does not lie within the uncertainty region.

EXMS B0820 - 424 — see above.

EXMS B0823 - 426 - see above. 
EXMS B0834 - 428 - probably G0834 - 430 and/or $4 \mathrm{U} 0836$ - 429; both were slewed over near the detection time though neither falls within the uncertainty region.

EXMS B0834+254 - Two extragalactic objects, Mr k1218 and B2 $0834+25$ lie within the uncertainty region but only $\mathrm{Mr} \mathrm{k} 1218$ is a cataloged X-ray emitter.

EXMS B0912+354 - 4C +34.31 lies within the uncertainty region, but is not a cataloged X-ray emitter.

EXMS B0917 - 549 - PKS 0916-54 lies within the uncertainty region, but is not a cataloged X-ray emitter.

EXMS B0923 - 308 - PKS 0923-30 lies within the uncertainty region, but is not a cataloged X-ray emitter. The proposed RASSBSC identification is near X-ray source 1H 0919-312.

EXMS B1042 - 599 - Eta Carinae lies within the uncertainty region, but is inconsistent with the position of the RASSBSC source.

EXMS B1044 - 595 — see above.

EXMS B1043 - 593 - see above.

EXMS B1040 - 593 - see above.

EXMS B1049+385 - B2 1049+38, a high-redshift radio galaxy (or possibly Seyfert 2), lies within the uncertainty region but is not a cataloged X-ray emitter.

EXMS B1123 - 588 — The proposed RASSBSC identification may be associated with the X-ray emitting SNR MSH11 - 54.

EXMS B1153+317 - 4C +31.38 lies within the uncertainty region but is not a cataloged X-ray emitter.

EXMS B1213+038 - The uncertainty region contains several radio sources, one of which $(4 \mathrm{C}+04.41)$ corresponds to X-ray source 1ES $1215+039$.

EXMS B1155 - 187 - Three obscure emission line galaxies lie within the uncertainty region.

EXMS B1235+708 - 4C +70.13 lies within the uncertainty region, but is not a cataloged X-ray emitter.

EXMS B1246 - 410B - ESO 323-G32 is a Seyfert 2 in cluster A3526.

EXMS B1254+276 - multiple unclassified AGN in Veron catalog. Multiple entries all have name UNKNOWN.

EXMS B1324 - 312 - A3558 is a cataloged X-ray emitter.

EXMS B1415+253 - NGC 5548 and 1E 14156+259 are two X-ray emitting AGN.

EXMS B1415+255 - see above.

EXMS B1416+256 - see above.

EXMS B1517 - 613 - TrA X-1 was slewed over near the detection time but would have appeared near the edge of the wrong quadrant.

EXMS B1525+525 - 4C +52.35 lies within the uncertainty region but is not a cataloged X-ray emitter.
EXMS B1550 - $609-4 \mathrm{U} 1543-624$ and 4U1556-605 were both slewed over near the detection time, but neither fell within the uncertainty region.

EXMS B1611 - 508 - HW Nor is within $10^{\prime}$ of 18 $\mathrm{X}$-ray sources.

EXMS B1637 - 671 - 4U1627-673 was slewed over near the time of detection but would have appeared in the wrong quadrant.

EXMS B1658 - 228A - an unidentified source less than a degree from another unidentified source, EXMS B1658 - 228B.

EXMS B1658 - 228B - see above.

EXMS B1702 - 429 - probably 4U1705-440 and/or $4 \mathrm{U} 1702-429$; both were slewed over near the detection time though neither falls within the uncertainty region.

EXMS B1719 - 436 - an unidentified source less than a degree from another unidentified source, EXMS B1722-442.

EXMS B1722 - 442 - see above.

EXMS B1721 - 231A - an unidentified source less than a degree from two other unidentified sources, EXMS B1721 - 231B and EXMS B1721 - 231C.

EXMS B1721 - 231B - see above.

EXMS B1721 - 231C — see above.

EXMS B1730 - 445 - 4U1735-444 passed slightly beyond the field of view near the time of the detection.

EXMS B1734 - 155 - TX Ser lies within the uncertainty region and is within $10^{\prime}$ of $1 \mathrm{RXS} \mathrm{J173735.1-152357}$ and $2 \mathrm{E} 1734.7-1522$.

EXMS B1741 - 337 - PKS 1742-337 lies within the uncertainty region, but is not a cataloged $\mathrm{X}$-ray emitter.

EXMS B1743 - 300A - probably GC X-1 or one of the many nearby objects, most of which were slewed over but none of which fell within the uncertainty region.

EXMS B1743 - 300B - see above.

EXMS B1743 - 303B - probably one or more of the many Galactic Center objects including SL1744-300 and neighboring sources, many of which were slewed over but none of which fell within the uncertainty region.

EXMS B1747 - 366 - probably 4U1746-360 and/or A1744-361; both were slewed over near the detection time though neither falls within the uncertainty region.

EXMS B1754 - 289 - an unidentified source less than a degree from another unidentified source, EXMS B1755-295.

EXMS B1755 - 295 - see above.

EXMS B1827 - 100 - an unidentified source less than a degree from another unidentified source, EXMS B1828100 .

EXMS B1828 - 100 - see above. 
EXMS B1837 - 052 - Near the SNR G27.4+0.0.

EXMS B1846 - 029A - In addition to EXO1846-03, G1845-03 and A1845-024 were also slewed over near the detection time but neither fell within the uncertainty region.

EXMS B1905+009 - Probably 4U1905+000 and/or Aql X-1, both of which were slewed over but neither of which fell within the uncertainty region. V810 Aql does lie within the uncertainty region and within $10^{\prime}$ of five Rosat sources.

EXMS B1906+007 - 4C +00.71 lies within the uncertainty region, but is not a cataloged X-ray emitter.

EXMS B1931+800 - 4C +79.20 lies within the uncertainty region, but is not a cataloged X-ray emitter.

EXMS B1948+113 - 4C +11.59 lies within the uncertainty region, but is not a cataloged X-ray emitter.

EXMS B1955+123 - an unidentified source less than a degree from 4 U1957+115, and also another unidentified source, EXMS B1955+124.

EXMS B1955+124 - see above.

EXMS B2124+751 - 4C +74.27 lies within uncertainty region, but is not a cataloged $\mathrm{X}$-ray emitter.

EXMS B2216 - 389 - Q2217-391, an extragalactic object, lies within the uncertainty region, but is not a cataloged X-ray emitter. 1H 2217-392 is a nearby stellar $\mathrm{X}$-ray source.

EXMS B2232 - 734 - PKS 2238-73 lies within the uncertainty region but is not a cataloged X-ray emitter.

EXMS B2233 - 657 - PKS 2239-65 lies within the uncertainty region, but is not a cataloged X-ray emitter.

EXMS B2223 - 331 - PKS 2224-33 lies within the uncertainty region, but is not a cataloged X-ray emitter.

\subsection{Systematic effects and accuracy of derived parameters}

In order to establish that the catalog is free of obvious systematic errors, a large subsample of sources was studied in detail. The adopted subsample contains 463 slew sources which were assigned identifications from the XRBCAT database of X-ray binaries (van Paradijs 1995), and where the identifications lie within the formal uncertainty regions $($ Flag $=4)$. Apart from the fact that this is the most numerous category of counterpart in the EXMS, these $\mathrm{X}$-ray binaries have well defined positions and tend to be bright X-ray sources. This helps ensure that they can be detected near the edge of the FOV. The disadvantage with this population is their strong concentration towards the galactic plane, and their intrinsic variability. The behavior of the offsets between these sources and the center of their uncertainty regions was simulated and studied, as is now discussed.

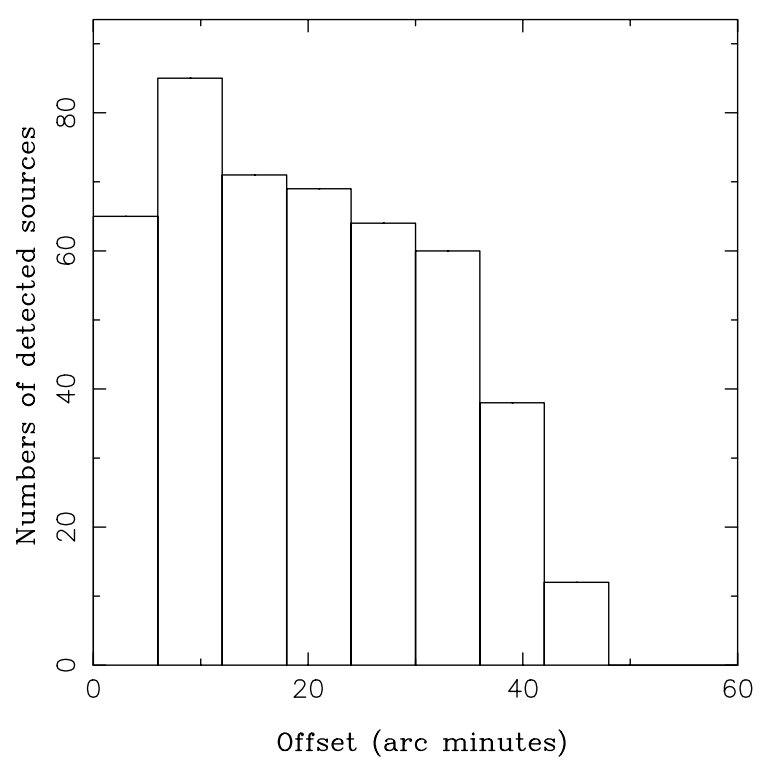

Fig. 10. The histogram of observed source offsets (in 6.0 bins) for the 463 sources identified as X-ray binaries whose positions are within the formal uncertainty regions

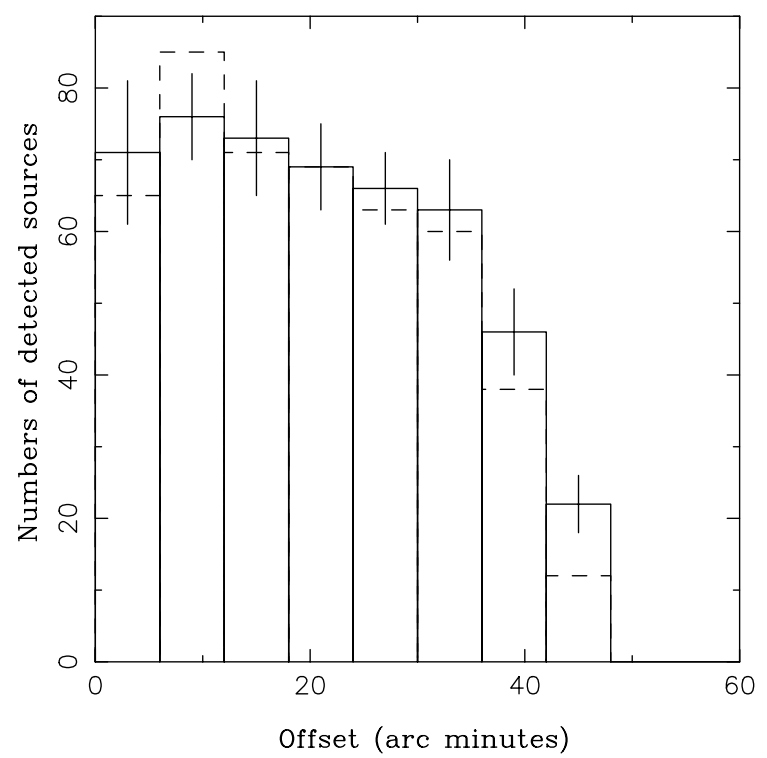

Fig. 11. A simulated histogram of source offsets (in 6. ${ }^{\prime} 0$ bins) for 500 sources, produced by averaging ten simulations. The variance in each bin is shown by a vertical line. The observed histogram (Fig. 10) is plotted using a broken line

\subsubsection{FOV offset simulation}

The offset of a given detection in the EXMS is simply the angular distance between the centroid of the uncertainty region and the catalog coordinates of the proposed identification. Since it is required that all identifications passed through the FOV, the maximum possible offset is given by the $45^{\prime} \mathrm{FWHM}$ of the collimator response. For simplicity the offsets are broken down into two components, defined in Fig. 7. The absolute offset for source $\mathrm{E}$ is the scalar 


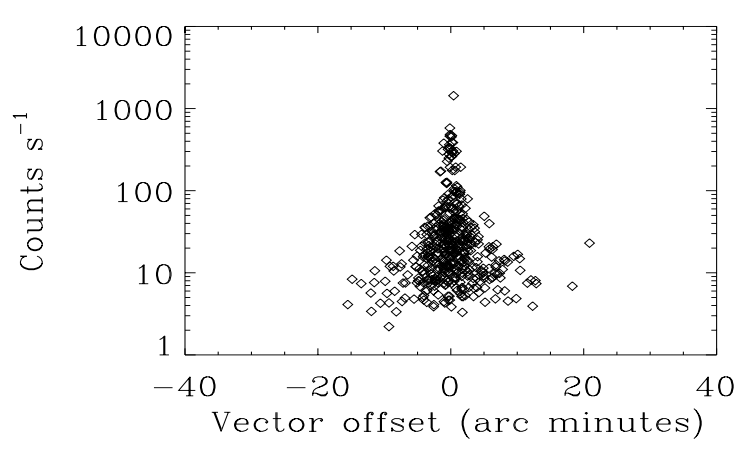

Fig. 12. Distribution of vector offsets (component of offset perpendicular to the direction of slew; vector EM in Fig. 7) for the 463 sources indentified with X-ray binaries which also lie within the formal uncertainty regions

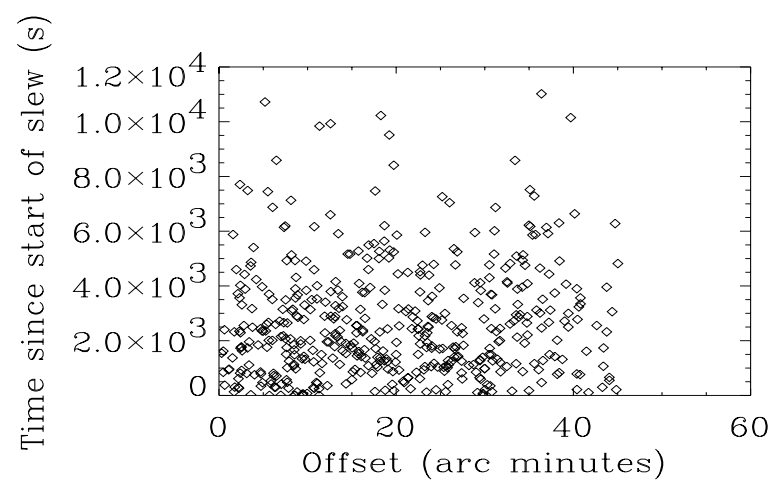

Fig. 13. Plot of absolute offsets (the distance EC in Fig. 7) against time since start of first slew leg, for the 463 sources indentified with X-ray binaries which also lie within their own error boxes. No time-dependent effects are evident

distance EC and the vector offset is EM. Similar offsets are defined for internal sources.

Absolute offsets were derived for the 463 XRBCAT identifications in the EXMS, and these were then summed into bins of $6{ }^{\prime} 0$, resulting in the histogram of Fig. 10. In order to understand the shape of this distribution, a simulation was performed in the following manner. First, a population of X-ray binaries was created with a realistic count rate distribution. This was achieved by correlating the XRBCAT against the ME database to obtain normalised ME counts for each matching source. Sources were then drawn randomly from this population and assigned random positions along a line, approximating the narrow uncertainty regions of the true sample. The observed counts of each source were then adjusted in accordance with the distance of the source from the center of the line, using the ME collimator response function. The average background count rate of the $1-8 \mathrm{keV}$ lightcurves is $\sim 15$ counts s$^{-1}$ half $^{-1}$, and this was adopted in the simulations. Since the background events are predominantly particle-induced, they are independent of

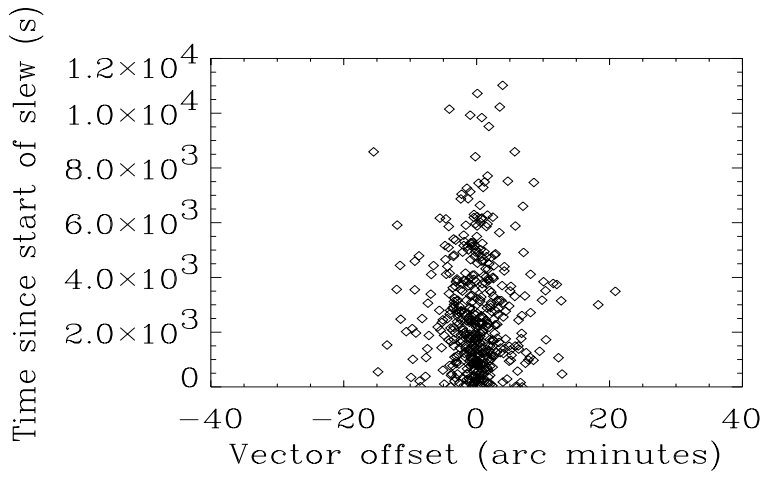

Fig. 14. Plot of vector offsets against time since start of first slew leg, for the same sample as in Fig. 12. Again no timedependent effects are evident

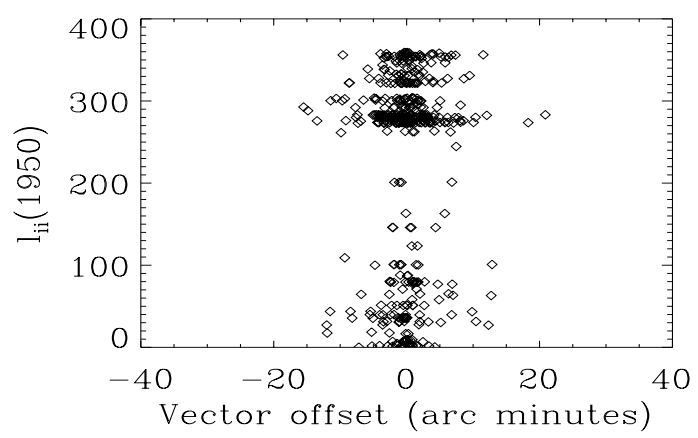

Fig. 15. Plot of vector offsets against galactic longitude, $l_{\mathrm{II}}$, demonstrating the lack of any dependence

collimator distance. As a consequence, faint sources are not detected unless they fall near the middle of the simulated FOV, but as such sources are more numerous, they play an important role in the shape of the offset histogram. The procedure was repeated until $\approx 460$ sources had been detected. Since there are $<100$ detections in each bin, the detailed shape of the histogram is dependent on statistical fluctuations. In order to characterize the shape and variance of a mean histogram, the results of ten independent simulations were averaged. The overall shape of the observed histogram is reproduced satisfactorily, with an approximately flat-topped distribution out to about 0.6 of the maximum offset (Fig. 11). In most bins, the real and simulated histograms agree within the variance of the simulation, and the small discrepancies that exist can be understood in terms of limitations in the simulation, particularly in the manner in which the detection threshold is modeled, and the fact that a few bright sources contribute predominantly to the detected numbers of sources in the high-offset bins.

Having established that the source offsets for this subsample are free of obvious systematic effects, the behavior of the vector component of the offsets which lie in the slew direction was examined. In the absence of 


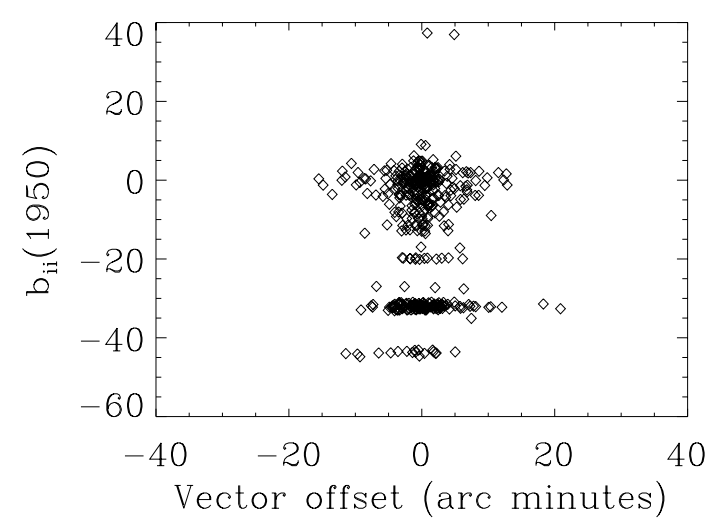

Fig. 16. As for Fig. 15, but for galactic latitude

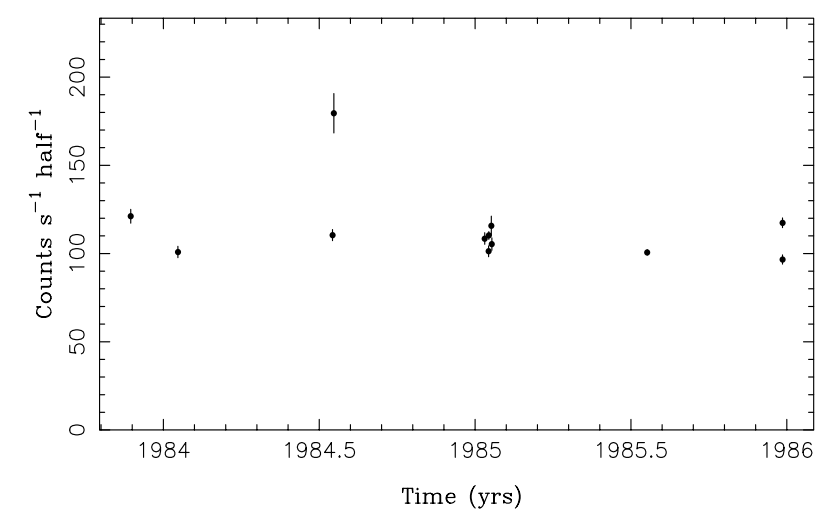

Fig. 17. EXMS collimator corrected count rates for the Cas-A SNR (assuming a point source). The expected count rate is 108 count s $^{-1}$ half $^{-1}$. With the exception of the measurement on $1984 / 201$, which occured at an offset of $35^{\prime}$, the count rates lie within $\sim 15 \%$ of the expected value

systematic errors in the pointing and timing information, the resultant distribution of offsets should be clustered symmetrically around zero. This is indeed the case, as shown in Fig. 12, where the 463 vector offsets are plotted against raw count rate. The mean vector offset is consistent with zero: $(-0.01 \pm 0.20)^{\prime}$. As is to be expected, the spread in the offsets is largest for the faintest sources.

The behavior of the absolute and vector offsets as a function of the elapsed time since the start of each slew was also examined, to ascertain whether the spacecraft attitude reconstruction gradually becomes less accurate. Since the slew durations vary from hundreds to thousands of seconds, the data span a wide time range, but it is clear (see Figs. 13 and 14) that the offsets appear random and independent of elapsed time.

Finally, the distributions of the absolute and vector offsets as a function of position in the sky were examined. Due to the sample being drawn solely from X-ray binaries, the sources are clustered around the Galactic plane and the Magellanic clouds. This means that there are large areas of the sky where the offsets are not sampled. However,

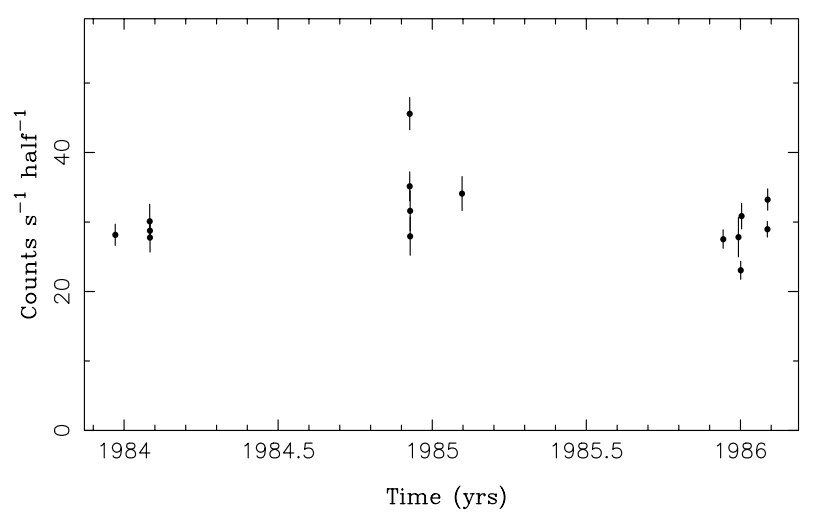

Fig. 18. Collimator corrected count rates for the Tycho SNR (assuming a point source). The expected count rate is 32 count $^{-1}$ half $^{-1}$, consistent with the mean EXMS value of $31 \pm 5$ count $^{-1}$ half $^{-1}$

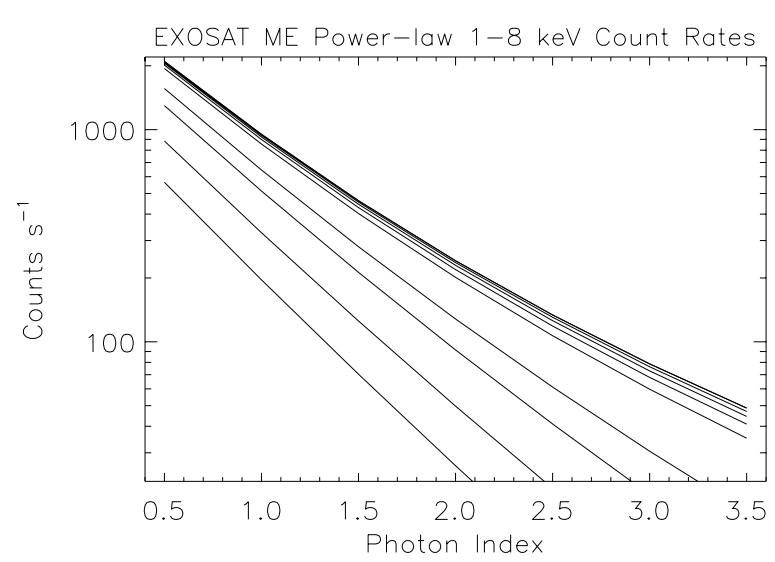

Fig. 19. Curves for converting from EXOSAT ME count rates to deadtime corrected fluxes for power-law spectra. From top to bottom, the curves give the predicted count rates for a spectral normalization of unity for $N_{\mathrm{H}}$ values of $<10^{21}, 10^{21}, 2.510^{21}$, $510^{21}, 10^{22}, 510^{22}, 10^{23}, 2.510^{23}$, and $510^{23}$ atoms $\mathrm{cm}^{-2}$

at least for the subsample, there is no suggestion of any position-dependent offset effects in either equatorial or Galactic coordinates (see Figs. 15 and 16 for the latter).

\subsubsection{Count rate accuracy}

Due to the variability of most X-ray sources, there are few sources in the EXMS which enable the accuracy of the derived count rates to be estimated. The brightest sources, such as X-ray binaries, are highly variable, while those sources which are time-invariant tend to be faint and extended (the latter leading to incorrectly estimated collimator corrections). Nevertheless, the observations of SNRs within the EXMS are in good agreement with the expected count rates based on ME pointed observations.

As an example, in 12 detections of Cas-A between 1983 and 1986, the derived EXMS count rates vary be-

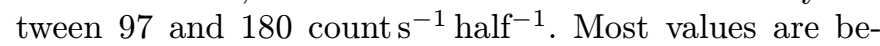
tween 100 and 120 count $^{-1}$ half $^{-1}$, with a mean value of 


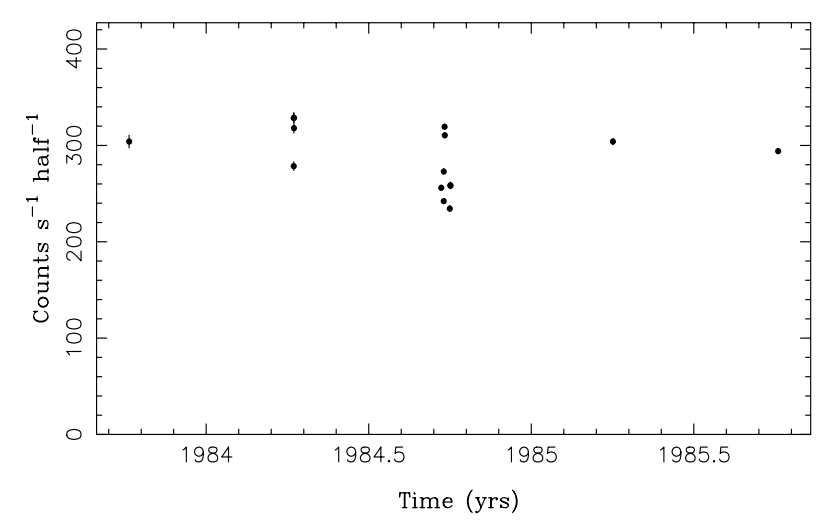

Fig. 20. EXMS detections of the XRB Ser X-1. Two pointed observations performed on $1985 / 251$ and 252 recorded count rates of 344 and 343 count $^{-1}$ half $^{-1}$, similar to the EXMS values

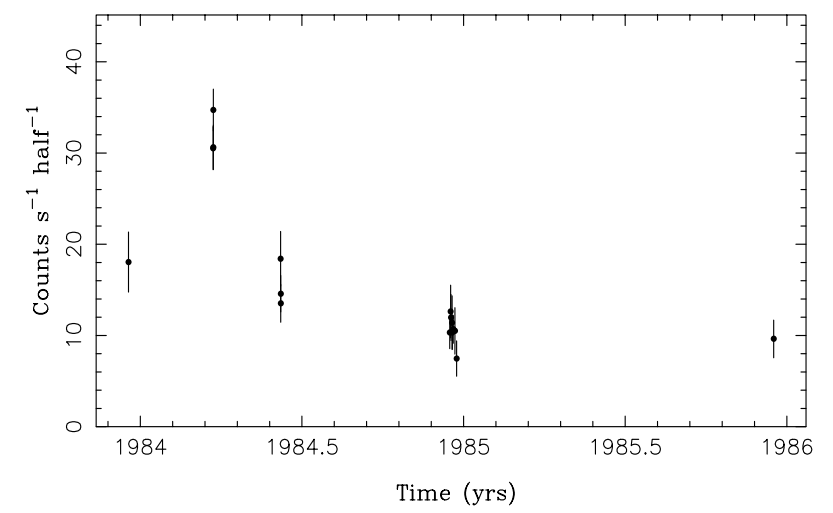

Fig. 21. EXMS detections of the XRB 4U 0918-549 showing an apparant long-term decrease in $1-8 \mathrm{keV}$ intensity

$114 \pm 9$ count $^{-1}$ half $^{-1}$ (see Fig. 17). The detection with the highest count rate is also that with the largest offset distance of $35^{\prime}$ (see Table 3 ). The extended nature of the source leads to an overestimated collimator correction. However, the mean value is in good agreement with the count rate during the single pointed ME observation of Cas-A of 108 count $\mathrm{s}^{-1}$ half $^{-1}$. Similar agreement is seen for the 15 slew detections of Tycho, with a mean value of $31 \pm$

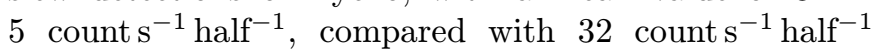
from two pointed observations (Fig. 18).

\subsubsection{Count rate to flux conversion}

An approximate conversion from count rate to flux may be obtained by noting that $1 \mathrm{ME}$ count $\mathrm{s}^{-1}$ half $^{-1}$ in the energy range $2-10 \mathrm{keV}$ is approximately equal to $1.210^{-11} \mathrm{erg} \mathrm{cm}^{-2} \mathrm{~s}^{-1}$. This conversion is dependent on spectral shape and curves for converting from the EXOSAT ME count rates given in Tables $3-5$ to $1-8 \mathrm{keV}$ fluxes are given in Fig. 19. This figure gives the conversion factors for a power-law model with photon indices between 0.5 and 3.5 and absorptions $<510^{23}$ atoms $\mathrm{cm}^{-2}$. The

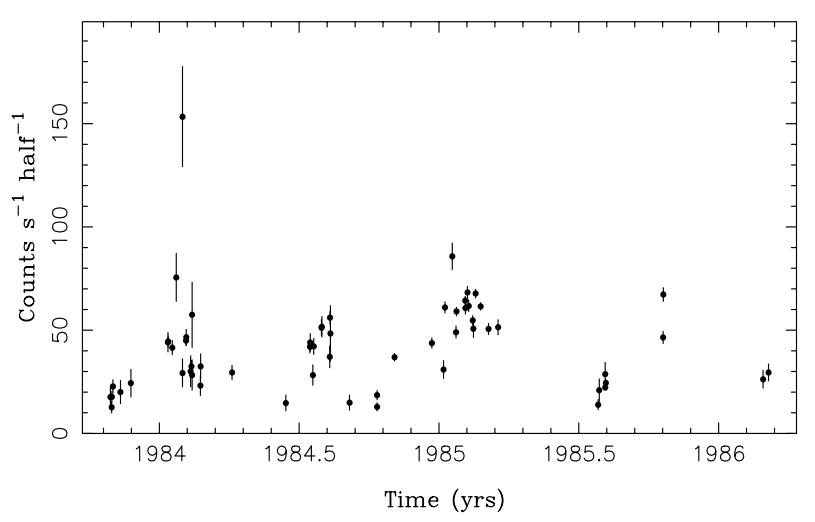

Fig. 22. EXMS detections of the XRB LMC X-3. The 198 (or possibly 99) day periodicity discovered by Cowley et al. (1991), may be evident

cataloged $1-8 \mathrm{keV}$ count rate, $c$, should first be corrected for deadtime losses by multiplying it by an approximate deadtime factor, $d_{\text {time }}=1.09+2.5610^{-4} c+5.610^{-8} c^{2}$. Comparison with the count rate read from Fig. 19 gives the power-law spectral normalization at $1 \mathrm{keV}$, and hence the flux.

\section{Summary}

The EXMS, a new catalog of X-ray detections derived from observations made by EXOSAT during slew manoeuvres between 1983 and 1986, is presented. Where possible, detections are identified with cataloged sources and the raw count rates corrected for collimator losses. Many types of object are detected, although X-ray binaries constitute the most common identification. The catalog has been shown to be free of obvious systematic errors and to contain reliable count rates for time-invariant sources. An electronic version of this catalog is maintained within the EXOSAT database and archive system at ESTEC (telnet://xray@exosat.estec.esa.nl).

The catalog contains new data on the long-term time variability of many well known sources and in particular on X-ray binaries. 95 different X-ray binaries appear in Table 3; roughly half of all X-ray binaries which have ever been detected. All of the expected X-ray binaries were seen, many of them repeatedly. We note that the transient source EXO 0748 - 676 appears as an EXMS source several times before it was actually detected in February 1985. Repeated source detections tend to be grouped at six monthly intervals due to the way in which EXOSAT slewed (see Sect. 1). For instance, Ser X-1 (Fig. 20) was detected on 15 occasions between 1983/279 and 1985/278, on nine separate days in five clusters each spaced by six months. Lightcurves for the XRBs 4U 0918-549 and LMC X-3 are presented in Figs. 21-22, together with brief comments. 
Acknowledgements. We thank H. Siddiqui, A. Hazell, K. Bennett and E. Kuulkers. F. Ochsenbein of the Observatoire Astronomique de Strasbourg is thanked for help on the EXMS naming policy. In addition to the online databases at ESTEC, this research made use of data obtained from the High Energy Astrophysics Science Archive Research Center, provided by the NASA-Goddard Space Flight Center. We thank the referee, Dr. W. Voges, for many helpful suggestions.

\section{References}

Cowley A.P., Schmidtke P.C., Ebisawa K., et al., 1991, ApJ 381,526

De Korte P., Bleeker J.A.M., den Boggende A.J.F., et al., 1981, Space Sci. Rev. 30, 495

Elvis M., Plummer D., Schachter J., Fabbiano G., 1992, ApJS 80,257

Forman W., Jones C., Cominsky L., et al., 1978, ApJS 38, 357 Gottwald M., 1984, EXOSAT Express 4, 46

Kholopov P.N., Samus N.N., Kazarovets B.V., Frolov M.S., Kireeva N.N., 1989, Inf. bull. Variable Stars 3323, 1

Kuulkers E., 1995, PhD thesis. University of Amsterdam

McHardy I.M., Lawrence A., Pye J.P., Pounds K.A., 1981,
MNRAS 197, 865

Peacock A., Andresen R.D., Manzo G., et al., 1981, Space Sci. Rev. 30, 525

Ritter H., 1990, A\&AS 85, 1179

Turner M.J.L., Smith A., Zimmermann H.U., 1981, Space Sci. Rev. 30, 512

Van Paradijs, J., 1995, A catalog of X-ray binaries. In: Lewin W.H.G., van Paradijs J., van den Heuvel E.P.J. (eds.). Cambridge Astrophysics Ser. 26, p. 536

Veron-Cetty M.P., Veron P., 1996, ESO Scientific Report 17

Voges W., Aschenbach B., Boller Th., et al., 1996, IAU Circ. 6420

Voges W., Aschenbach B., Boller Th., et al., 1998, A\&AS (in preparation)

Warwick R.S., Turner M.J.L., Watson M.G., Willingale R., 1985, Nat 317, 21

Warwick R.S., Norton A.J., Turner M.J.L., Watson M.G., Willingale R., 1988, MNRAS 232, 551

Warwick R.S., Marshall N., Fraser G.W., et al., 1981, MNRAS 197, 893

White N.E., Peacock A., 1988, Mem. della Società Astr. Ital. 59,7

Wood K.S., Meekins J.F., Yentis D.J., et al., 1984, ApJS 56, 507 